\title{
Impact of free tumor clusters on prognosis after resection of pulmonary adenocarcinoma
}

\author{
Junichi Morimoto, $\mathrm{MD}, \mathrm{PhD},{ }^{\mathrm{a}}$ Takahiro Nakajima, $\mathrm{MD}, \mathrm{PhD},{ }^{\mathrm{a}}$ Hidemi Suzuki, $\mathrm{MD}, \mathrm{PhD},{ }^{\mathrm{a}}$ \\ Kaoru Nagato, $\mathrm{MD}, \mathrm{PhD},{ }^{\mathrm{a}}$ Takekazu Iwata, $\mathrm{MD}, \mathrm{PhD},{ }^{a}$ Shigetoshi Yoshida, $\mathrm{MD}, \mathrm{PhD},{ }^{\mathrm{a}}$ \\ Masaki Fukuyo, $\mathrm{PhD},{ }^{\mathrm{b}}$ Satoshi Ota, $\mathrm{MD}, \mathrm{PhD},{ }^{\mathrm{c}}$ Yukio Nakatani, $\mathrm{MD}, \mathrm{PhD},{ }^{\mathrm{c}}$ and Ichiro Yoshino, $\mathrm{MD}, \mathrm{PhD}^{\mathrm{a}}$
}

\begin{abstract}
Objective: Pulmonary adenocarcinoma with a micropapillary component (MPC) has aggressive malignant behavior even if resectable. The aim of this study was to determine clinicopathologic features of patients who underwent surgery for pulmonary adenocarcinoma harboring MPCs, with particular focus on coexistent free tumor clusters (FTCs).

Methods: We retrospectively reviewed 444 patients with pulmonary adenocarcinoma who underwent surgery from March 2007 to July 2013. An MPC was defined as a $>5 \%$ micropapillary pattern. We also defined FTCs to be a group of more than 3 small clusters containing $<20$ nonintegrated micropapillary tumor cells that were spreading within air spaces, $>3 \mathrm{~mm}$ apart from the main tumor. The clinicopathologic characteristics of patients with and without FTCs were retrospectively investigated in MPC-positive patients.
\end{abstract}

Results: MPCs were identified in 67 patients (15.1\%), 31 of whom (46.3\%) were positive for FTCs. The distance between the furthest edge of FTCs and main tumors did not exceed the diameter of the main tumor in each case (average, $7.3 \mathrm{~mm}$ ). Locoregional recurrences were frequently observed in FTC-positive patients. FTC-positive patients experienced a significantly lower 5-year recurrence-free survival rate compared with FTC-negative/MPC-positive patients $(20.4 \%$ vs $52.2 \%, \quad P<.001)$. Recurrence-free survival of FTC-negative and -positive patients was equivalent to that of patients with p-T2 and p-T3 MPC-negative adenocarcinoma, respectively.

Conclusions: Coexistence of FTCs resulted in a further negative impact on postoperative prognosis among MPC-positive adenocarcinomas and should be considered for upstaging the $\mathrm{p}-\mathrm{T}$ factor and during evaluation of surgical margins. (J Thorac Cardiovasc Surg 2016;152:64-72)

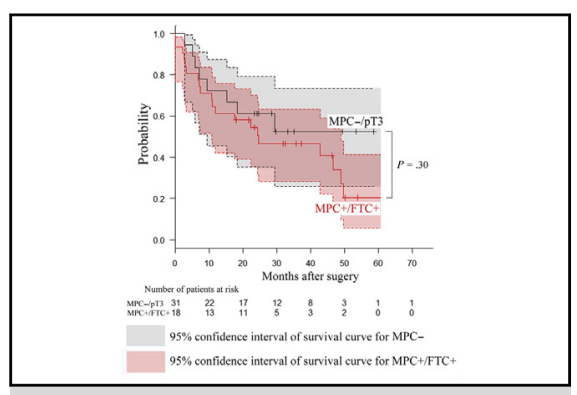

FTC-positive patients had equivalent disease-free survival to $\mathrm{p}$-T3 MPC-negative patients.

\section{Central Message}

Existence of free tumor clusters resulted in negative impact on postoperative prognosis among micropapillary positive adenocarcinomas.

\section{Perspective}

To achieve curative resection in free tumor cluster -positive adenocarcinoma, the resection margin should be wider and wedge resection is not recommended. Recurrence-free survival of FTC-negative and -positive patients were equivalent to that of patients with $\mathrm{p}-\mathrm{T} 2$ and p-T3 nonmicropapillary adenocarcinoma, respectively. Upstaging of $\mathrm{p}-\mathrm{T}$ factor should be considered in this population.

See Editorial Commentary page 73 .
Recently, invasive lung adenocarcinomas have been classified into 5 subtypes according to their predominant components: papillary, lepidic, acinar, solid, or micropapillary. ${ }^{1} \mathrm{Of}$

From the Departments of General Thoracic Surgery, ${ }^{\mathrm{a}}$ Molecular Oncology, ${ }^{\mathrm{b}}$ and Diagnostic Pathology, ${ }^{\mathrm{c}}$ Chiba University Graduate School of Medicine, Chiba, Japan.

This work was supported by Grant-in-Aid for Innovative Therapeutics Project in Chiba University (to T.N.).

Received for publication July 28, 2015; revisions received Jan 4, 2016; accepted for publication March 13, 2016.

Address for reprints: Takahiro Nakajima, MD, PhD, Department of General Thoracic Surgery, Chiba University Graduate School of Medicine 1-8-1 Inohana, Chiba 260-8670, Japan (E-mail: takahiro_nakajima@med.miyazaki-u.ac.jp). 0022-5223/\$36.00

Copyright $₫ 2016$ Published by Elsevier Inc. on behalf of The American Association for Thoracic Surgery

http://dx.doi.org/10.1016/j.jtcvs.2016.03.088 these, micropapillary-predominant type adenocarcinoma has been reported to be an indicator of poor prognosis. ${ }^{2}$ Micropapillary-predominant type tumors are known to be more invasive and more likely to metastasize compared with other subtypes. ${ }^{2}$ Zhang et $\mathrm{al}^{3}$ analyzed stage I adenocarcinomas and the prognostic impact of pathologic subtype as "high histologic grade," including

Scanning this QR code will take you to supplemental figure and table for this article. 


\section{Abbreviations and Acronyms \\ $\mathrm{CI}=$ confidence interval \\ FTC $=$ free tumor cluster \\ MPC $=$ micropapillary component \\ $\mathrm{RR}=$ relative risk \\ STAS $=$ tumor spread through air spaces}

micropapillary-predominant adenocarcinoma. These investigators reported that high histologic grade adenocarcinoma was associated with significantly shorter 5-year recurrencefree survival compared with low histologic grade adenocarcinoma $(P<.001)$. Micropapillary components (MPCs) in pulmonary adenocarcinoma display morphologic features such as small papillary tufts with no fibrovascular core lying freely in the alveolar spaces or encased within connective tissue, suggesting a unique growth pattern. ${ }^{2}$ In addition to micropapillary-predominant adenocarcinoma, tumors that contain even a small MPC have been reported to also show aggressive behavior. ${ }^{4}$ Cha et $\mathrm{al}^{5}$ analyzed the prognosis in adenocarcinoma that contains a $>1 \%$ MPC. These researchers reported that patients with MPCs experienced a shorter disease-free survival and overall survival (hazard ratio, 4.17; 95\% confidence interval [CI], 1.28-13.63).

Another unique characteristic of some MPCs is the presence of nonintegrated free tumor clusters (FTCs) located apart from the main tumors. ${ }^{6}$ Kadota et $\mathrm{al}^{7}$ defined the tumor spread through air spaces (STAS) as tumor cells with micropapillary structures, solid nests, or single cells spreading within air spaces in the lung parenchyma beyond the edge of the main tumor. They reported that the presence of STAS is a significant risk factor of recurrence in small lung adenocarcinomas treated with limited resection. ${ }^{7}$ Recently, a unique clinicopathologic characteristic of STAS was reported by Warth et $\mathrm{al}^{8}{ }^{8}$ and the STAS morphologic character itself was shown to be a poor prognostic factor as well as a risk for metastases. The FTC does not contain solid nests or single cells and is strictly defined as MPCs spreading within air spaces; hence, it was encompassed in the STAS. The clinical impact of coexisting FTCs in pulmonary adenocarcinoma with a micropapillary pattern remains unknown. The purpose of this retrospective study was to determine the clinicopathologic features of pulmonary adenocarcinomas with MPCs that harbor a $>5 \%$ micropapillary pattern, with particular focus on the clinical impact of existing FTCs in patients who have undergone surgery.

\section{METHODS \\ Patients}

All clinical and pathologic data were retrieved from the medical records of 478 patients who underwent surgery for lung adenocarcinoma from March 2007 to July 2013 at Chiba University Hospital. Among these, 34 patients were excluded because of situations that made histologic reevaluation difficult, such as preoperative chemotherapy or obstructive pneumonia. Therefore, the patients who underwent induction treatmen were excluded from this analysis. The remaining 444 patients with adenocarcinoma were evaluated. Tumors were classified by histologic subtype according to the World Health Organization classification. ${ }^{9}$ Staging was performed according to the seventh edition of the TNM Classification. ${ }^{10}$ All patients underwent follow-up at our outpatient clinic after surgery. The ethics committee at Chiba University approved this study (No. 1991), and written consent was waived because of the retrospective nature of this chart review.

\section{Pathologic Evaluation}

We reevaluated formalin-fixed, paraffin-embedded 4- $\mu \mathrm{m}$ sections with hematoxylin-eosin staining. The sections were re-reviewed by independent board-certified pathologists (S.O. and Y.N.). The definition of micropapillary clusters is from a previous report. ${ }^{9}$ An MPC was defined as a $>5 \%$ micropapillary pattern. To avoid artifacts made during the pathologic sectioning process, we defined more than 3 clusters as true FTCs. We also defined FTCs as a group of more than 3 small clusters containing $<20$ nonintegrated micropapillary tumor cells in each cluster that was floating in an alveolar space $>3 \mathrm{~mm}$ away from the main tumor margin. The pathologic diagnosis was made following these criteria and the reproducibility of the diagnosis of FTC and MPC was high in this study. When the diagnoses differed between the pathologists, which happened rarely, the final diagnosis was made after discussions until reaching a consensus.

\section{Statistical Analysis}

Comparisons between categorical data of 2 patient groups were performed using a 2-sided Mann-Whitney $U$ test and $\chi^{2}$ test. A $P$ value $<.05$ was deemed significant. Correlation between 2 independent values was analyzed by the Spearman test. The survival analysis was calculated using the Kaplan-Meier method, and differences were determined using log-rank analysis.

All statistical analyses were performed with EZR (Saitama Medical Center, Jichi Medical University, Saitama, Japan), which is a graphical user interface for R (The R Foundation for Statistical Computing, Vienna, Austria).

\section{RESULTS \\ Patient Characteristics}

Overall, 444 patients with pulmonary adenocarcinoma who underwent surgery met the criteria for this analysis. Among these 444 patients, $67(15.1 \%)$ had MPCs and 31 of these 67 patients $(46.3 \%)$ had FTCs in their adenocarcinoma histology. Patient characteristics are shown in Table 1. No significant differences were observed between patients without MPCs (control) and those with MPCs with respect to age, gender, or background comorbidities. Most of the MPC-positive patients underwent anatomic resection for lung cancer, and only 1 patient underwent wedge resection. More patients with MPCs received adjuvant treatment because of the advanced pathologic stage. Patients with MPCs had a significantly higher incidence of pathologic nodal metastasis (pathologic nodal metastasis positive; $40.3 \%$ vs $12.7 \%$; relative risk [RR]: 0.316 ; 95\% CI, 0.213-0.468), invasion into the lymphatic system (pathologic lymphatic invasion positive; $43.3 \%$ vs $9.0 \%$; RR, $0.208 ; 95 \%$ CI, 0.137-0.318), invasion into blood vessels (pathologic vascular invasion positive; $43.3 \%$ vs $21.8 \%$; RR, $0.503 ; 95 \% \quad \mathrm{CI}$, 0.360-0.702), and visceral pleural invasion (pathologic 
TABLE 1. Characteristics of micropapillary component-negative (gray group) and micropapillary component-positive (yellow group) patients

\begin{tabular}{|c|c|c|c|}
\hline & MPC - (gray group) & MPC + (yellow group) & \\
\hline Total $(n=444)$ & $(\mathbf{n}=377)$ & $(n=67)$ & $P$ value \\
\hline Age, y (range) & $66.6(36-87)$ & $66.1(42-85)$ & .21 \\
\hline Gender, male/female & $220(58.4 \%) / 157(41.6 \%)$ & $41(61.2 \%) / 26(38.8 \%)$ & .76 \\
\hline Surgical procedure & & & .017 \\
\hline Pneumonectomy & $1(0.3 \%)$ & $1(1.5 \%)$ & \\
\hline Lobectomy & $261(69.2 \%)$ & $56(83.6 \%)$ & \\
\hline Segmentectomy & $84(22.3 \%)$ & $9(13.4 \%)$ & \\
\hline Wedge resection & $31(8.2 \%)$ & $1(1.5 \%)$ & \\
\hline \multicolumn{4}{|l|}{ Clinical stage } \\
\hline T1/T2/T3/T4 & $280(74.3 \%) / 78(20.7 \%) / 16(4.2 \%) / 3(0.8 \%)$ & $44(65.7 \%) / 21(31.3 \%) / 2(3.0 \%) / 0$ & .24 \\
\hline $\mathrm{N} 0 / \mathrm{N} 1 / \mathrm{N} 2 / \mathrm{N} 3$ & $358(95.0 \%) / 15(4.0 \%) / 4(1.1 \%) / 0$ & $60(89.6 \%) / 5(7.5 \%) / 2(3.0 \%) / 0$ & .16 \\
\hline Stage I/II/III/IV & $338(89.7 \%) / 31(8.2 \%) / 8(2.1 \%) / 0$ & $55(82.1 \%) / 9(13.4 \%) / 3(4.5 \%) / 0$ & .19 \\
\hline \multicolumn{4}{|l|}{ Pathologic stage } \\
\hline T1/T2/T3/T4 & $305 / 40 / 28 / 4$ & $33 / 6 / 25 / 3$ & $<.0001$ \\
\hline $\mathrm{N} 0 / \mathrm{N} 1 / \mathrm{N} 2 / \mathrm{N} 3$ & $329 / 26 / 22 / 0$ & $39 / 5 / 22 / 0$ & $<.0001$ \\
\hline Pathologic lymphatic invasion & $34(9.0 \%)$ & $29(43.3 \%)$ & $<.0001$ \\
\hline Pathologic vascular invasion & $82(21.8 \%)$ & $29(43.3 \%)$ & $<.001$ \\
\hline Pathologic pleural invasion & $69(18.3 \%)$ & $26(38.9 \%)$ & $<.001$ \\
\hline Stage I/II/III/IV & $305(80.9 \%) / 40(10.6 \%) / 28(7.4 \%) / 4(1.1 \%)$ & $33(49.3 \%) / 6(9.0 \%) / 25(37.3 \%) / 3(4.5 \%)$ & $<.0001$ \\
\hline \multicolumn{4}{|l|}{ Comorbidities } \\
\hline $\begin{array}{l}\text { Preoperative forced expiratory } \\
\text { volume in } 1 \text { second }(\mathrm{L})\end{array}$ & 75.9 & 74.3 & .34 \\
\hline Smoking index (pack year) & 25.3 & 24.6 & .2 \\
\hline Chronic obstructive pulmonary disease & $81(21.5 \%)$ & $17(25.4 \%)$ & .58 \\
\hline Cardiac disorder & $67(17.8 \%)$ & $7(10.4 \%)$ & .13 \\
\hline Hypertension & $143(37.9 \%)$ & $32(47.8 \%)$ & .13 \\
\hline Diabetes & $49(13.0 \%)$ & $5(7.5 \%)$ & .28 \\
\hline $\begin{array}{l}\text { Adjuvant treatment (chemotherapy } \\
\text { and/or radiotherapy) }\end{array}$ & $72(19.1 \%)$ & $24(35.8 \%)$ & $<.05$ \\
\hline Recurrence of the disease & $78(20.7 \%)$ & $34(50.7 \%)$ & $<.0001$ \\
\hline Mortality during follow-up & $36(9.5 \%)$ & $21(31.3 \%)$ & $<.0001$ \\
\hline
\end{tabular}

pleural invasion positive; $38.9 \%$ vs $18.3 \%$; RR, 0.472 ; 95\% CI, 0.326-0.682) compared with patients without MPCs. No correlation was observed between the pattern of failure and the type of operation.

\section{FTCs}

The characteristics of patients with FTC-positive and FTC-negative disease are separately shown in Table 2. Patients with FTCs had a significantly higher incidence of invasion into the lymphatic system (pathologic lymphatic invasion positive; $P<.0001$ ) and invasion into blood vessels (pathologic vascular invasion positive; $P<.0001$ ) compared with patients without FTCs. The distance between FTCs and the surgical margin in the MPC-positive/FTC-positive group was shorter than the distance between tumors and the surgical margin in the MPC-positive/FTC-negative group $(19.6 \pm 2.2 \mathrm{~mm}$ vs $27.9 \pm 1.8 \mathrm{~mm} ; P<.001)$. A representative histologic image of FTCs is shown in Figure 1. Several small clusters are observed around the main tumor. A discrepancy in the sizes of tumor cells existing in the area between the macroscopic and microscopic views was noted (Figure 2). The mean distance between the furthest edges of FTCs from the margin of the main tumor was $7.3 \pm 0.8 \mathrm{~mm}$. The FTC distances and the diameter of the main tumors were not statistically correlated ( $r=0.17, P=.301)$, because the FTCs were located within a distance that did not exceed the diameters of the main tumors (Figure 3). Characteristics of FTC-positive patients were not significantly different from those of FTC-negative patients (Table 2).

\section{Recurrence-Free Survival}

Recurrence-free survival of MPC-positive and MPC-negative patients, and that of MPC-positive/FTCnegative and MPC-positive/FTC-positive patients, are shown in Figure 4, $A$ and $B$. The median follow-up period for the recurrence-free survival was 39.2 months and the median follow-up period for each population was 41.8 months for MPC-negative patients, 22.3 months for FTC-positive patients, and 32.5 months for FTC-negative 
TABLE 2. Characteristics of micropapillary component-positive/free tumor cluster-positive (red group) and micropapillary component-positive/ free tumor cluster-negative (green group) patients

\begin{tabular}{|c|c|c|c|}
\hline & MPC $+/ F T C+($ red group $)$ & MPC $+/$ FTC - (green group) & \\
\hline Total $(n=67)$ & $(\mathbf{n}=\mathbf{3 1})$ & $(\mathbf{n}=36)$ & $P$ value \\
\hline Age, y (range) & $67.5(51-77)$ & $64.9(42-85)$ & .27 \\
\hline Gender, male/female & $22(71.0 \%) / 9(29.0 \%)$ & $19(52.8 \%) / 17(47.2 \%)$ & .93 \\
\hline Surgical procedure & & & 1 \\
\hline Pneumonectomy & $0(0.0 \%)$ & $1(2.8 \%)$ & \\
\hline Lobectomy & $27(87.1 \%)$ & $29(80.6 \%)$ & \\
\hline Segmentectomy & $4(12.9 \%)$ & $5(13.9 \%)$ & \\
\hline Wedge resection & $0(0.0 \%)$ & $1(2.8 \%)$ & \\
\hline \multicolumn{4}{|l|}{ Pathologic stage } \\
\hline $\mathrm{T} 1 / \mathrm{T} 2 / \mathrm{T} 3 / \mathrm{T} 4$ & $14(45.2 \%) / 15(48.4 \%) / 2(6.5 \%) / 0(0.0 \%)$ & $14(38.9 \%) / 21(58.3 \%) / 1(2.8 \%) / 0(0.0 \%)$ & .64 \\
\hline $\mathrm{N} 0 / \mathrm{N} 1 / \mathrm{N} 2 / \mathrm{N} 3$ & $16(51.6 \%) / 2(6.5 \%) / 13(41.9 \%) / 0(0.0 \%)$ & $24(66.7 \%) / 3(8.3 \%) / 9(25.0 \%) / 0(0.0 \%)$ & .35 \\
\hline Pathologic lymphatic invasion & $37(9.8 \%)$ & $28(41.8 \%)$ & $<.0001$ \\
\hline Pathologic vascular invasion & $82(21.8 \%)$ & $29(43.3 \%)$ & $<.0001$ \\
\hline Pathologic pleural invasion & $14(45.2 \%)$ & $12(33.3 \%)$ & .32 \\
\hline Stage I/II/III/IV & $11(35.5 \%) / 3(9.7 \%) / 15(48.4 \%) / 2(6.5 \%)$ & $22(61.1 \%) / 3(8.3 \%) / 10(27.8 \%) / 1(2.8 \%)$ & .18 \\
\hline \multicolumn{4}{|l|}{ Tumor factors } \\
\hline Mean tumor diameter (mm) & $30.0 \pm 2.6$ & $30.0 \pm 2.9$ & .74 \\
\hline $\begin{array}{l}\text { Mean distance between tumor } \\
\text { and surgical margin }(\mathrm{mm})\end{array}$ & $26.0 \pm 2.2$ & $27.9 \pm 1.8$ & .51 \\
\hline $\begin{array}{l}\text { Mean distance between FTC } \\
\text { and main tumor }(\mathrm{mm})\end{array}$ & $7.3 \pm 0.8$ & NA & NA \\
\hline $\begin{array}{l}\text { Mean distance between FTC } \\
\text { and surgical margin (mm) }\end{array}$ & $19.6 \pm 2.2$ & NA & NA \\
\hline \multicolumn{4}{|l|}{ Nodal factors } \\
\hline Number of sampled nodal stations & $7.5 \pm 0.4$ & $7.6 \pm 0.3$ & .32 \\
\hline $\begin{array}{l}\text { Number of metastatic lymph } \\
\text { node stations }\end{array}$ & $3.5 \pm 0.5$ & $2.3 \pm 0.5$ & .2 \\
\hline \multicolumn{4}{|l|}{ Comorbidities } \\
\hline $\begin{array}{l}\text { Preoperative forced expiratory } \\
\text { volume in } 1 \text { second }\end{array}$ & 0.734 & 0.752 & .32 \\
\hline Smoking index & 605 & 392.8 & .15 \\
\hline Chronic obstructive pulmonary disease & $10(32.3 \%)$ & $8(22.2 \%)$ & .36 \\
\hline Cardiac disorder & $0(0.0 \%)$ & $7(19.4 \%)$ & .028 \\
\hline Hypertension & $13(41.9 \%)$ & $19(52.8 \%)$ & .38 \\
\hline Diabetes & $2(6.5 \%)$ & $4(11.1 \%)$ & .51 \\
\hline Adjuvant chemotherapy & $13(41.9 \%)$ & $11(30.6 \%)$ & .33 \\
\hline Recurrence of the disease & $20(64.5 \%)$ & $14(38.9 \%)$ & .015 \\
\hline Mortality during the follow-up & $13(41.9 \%)$ & $8(22.2 \%)$ & .048 \\
\hline
\end{tabular}

Red and green groups: The recurrence-free survival and overall survival of each group are shown in Figure 4, $B$, and Figure E1, $B$, respectively. $M P C$, Micropapillary component; FTC, nonintegrated free tumor cluster.

patients. The 5-year recurrence-free survival rates after surgery for these groups were $76.2 \% \quad(95 \% \quad \mathrm{CI}$, $70.7 \%-80.8 \%), 52.2 \%$ (95\% CI, 31.4\%-69.5\%), and 20.4\% (95\% CI, 5.8\%-41.2\%), respectively (Figure 4, B).

Multivariate analysis of the covariates, including age, surgical procedure (lobectomy or limited surgery), pathologic stage (stage I or higher), adjuvant therapy (yes or no), and existence of MPCs (positive or negative), was performed (Table 3). The existence of MPC remains a strong prognostic factor for recurrence-free survival (hazard ratio, 2.70; 95\% $\mathrm{CI}, 1.75-4.17 ; P<.0001)$.

Among MPC-positive patients, both FTC-positive and -negative patients experienced poorer survival rates than did patients without MPCs $(P=.016, P<.0001$, respectively). FTC-positive patients showed significantly worse survival compared with FTC-negative patients $(P=.015)$ (Figure $4, B)$. The confidence limits of each survival curve were shown in Table E1. We performed multivariate analysis of the covariates, including age, surgical procedure (lobectomy or limited surgery), pathologic stage (stage I or higher), adjuvant therapy (yes or no), distance to surgical margin (more or less than the tumor diameter), and existence of FTCs (positive or negative). Pathologic stage and the existence of FTCs were identified as independent prognostic factors for recurrence-free survival (Table 4). 


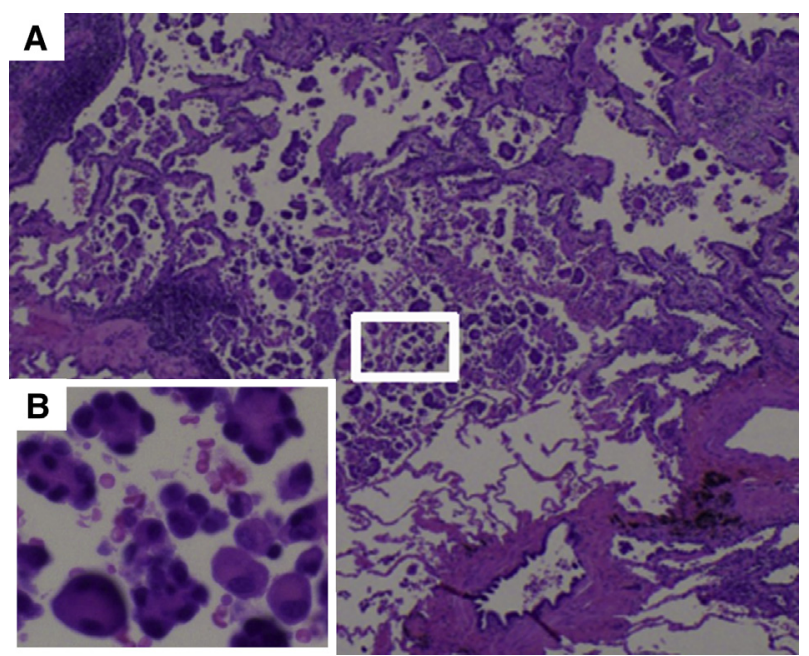

FIGURE 1. Pathologic findings of free tumor clusters in adenocarcinoma with micropapillary components. A, Representative pathologic findings of a micropapillary component (MPC)-positive pulmonary adenocarcinoma (low-power view). B, Individual clusters comprise 5 to 20 tumor cells with micropapillary features such as eccentric nuclei and absence of a central fibrovascular core (high-power view).

When MPC-negative patients were classified by $\mathrm{T}$ category, MPC-positive/FTC-positive and MPC-positive/ FTC-negative patients had disease-free survival curves equivalent to those of pathologic (p-)T2 and (p-)T3 MPC-negative patients, respectively (Figure 4, $C$ and $D$ ).

When the type of recurrence was analyzed in patients with MPC, FTC-positive patients tended to have a higher incidence of recurrence $(P=.036)$; however, the difference was not significant between FTC-positive and -negative patients (data not shown). We also analyzed the time-dependent process of local and distant recurrence, and the FTC-positive population showed local recurrence in the earlier observation period than distant metastasis; however, this difference was not significant because of the limited number of patients (data not shown).

\section{Overall Survival}

The median follow-up period for overall survival was 43.4 months and the median follow-up period for each population was 31.9 months for MPC-negative patients, 28.5 months for FTC-positive patients, and 34.5 months for FTC-negative patients. MPC-positive patients experienced shorter overall survival compared with MPC-negative patients (Figure E1, $A ; P<.0001$ ). In addition, MPC-positive/FTC-positive patients also experienced shorter overall survival compared with MPC-positive/FTC-negative patients (Figure E1, $B$; $P=.048$ ). The 5-year overall survival rates for MPCnegative, MPC-positive/FTC-negative, and MPC-positive/ FTC-positive patients were $86.3 \%$ (95\% CI, 0.809-0.903), $69.6 \%$ (95\% CI, $0.458-0.845)$, and $54.4 \%$ (95\% CI, $0.312-0.727)$, respectively.

\section{DISCUSSION}

Pulmonary adenocarcinoma with MPC is an aggressive disease that metastasizes to lymph nodes even at an early clinical stage, and has an unfavorable prognosis. ${ }^{4}$ In this study, we focused on the coexistence of FTCs that are a distinctive morphologic feature of MPC, and found that the coexistence of FTCs was an indicator of more aggressive clinical behavior among MPC-positive lung adenocarcinomas. Kadota et $\mathrm{al}^{7}$ reported that a similar phenomenon, which was described as STAS, was observed in $38 \%$ of patients with adenocarcinoma. STAS was defined as a tumor spread through air spaces with micropapillary structures, solid nests, or single cells spreading within air spaces in the lung parenchyma beyond the edge of the main tumor; hence, this study was not limited to the

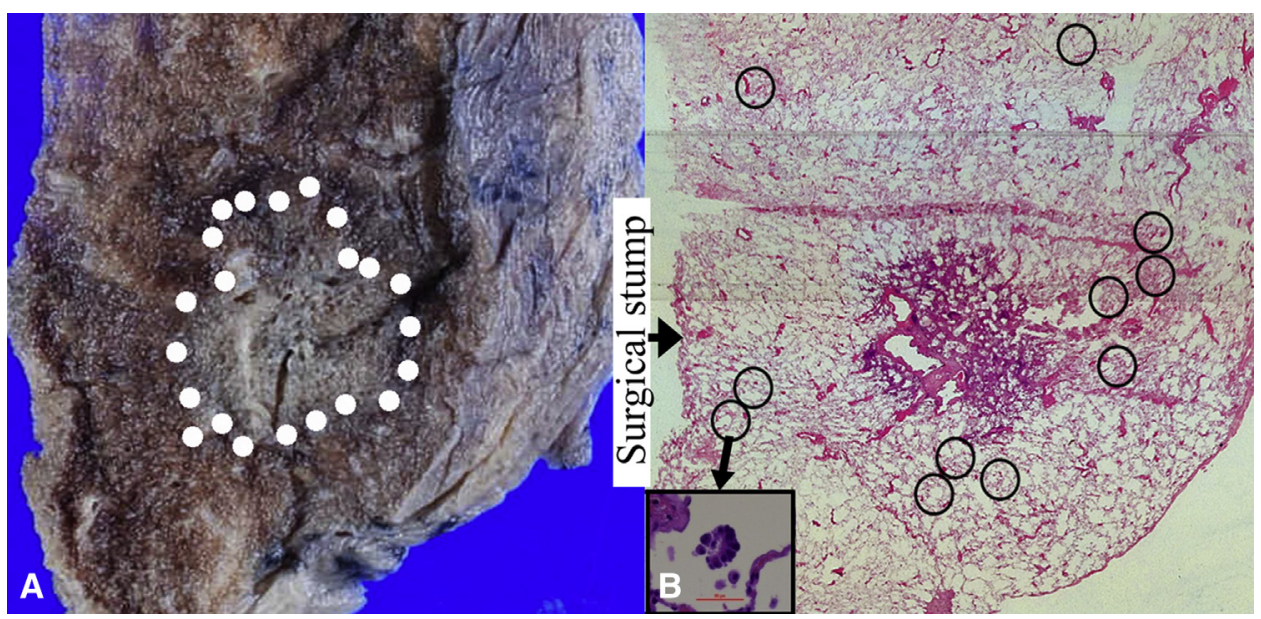

FIGURE 2. A discrepancy in the sizes of tumor cells existing in the area between the macroscopic and microscopic views. A, The gross section of the tumor measured $24 \mathrm{~mm}$ (dotted line) and showed a 15-mm tumor with a sufficient surgical margin. B, Several nonintegrated free tumor clusters were observed floating in the alveolar spaces (circle). In this case, the cluster was found only $3 \mathrm{~mm}$ away from the surgical margin. 


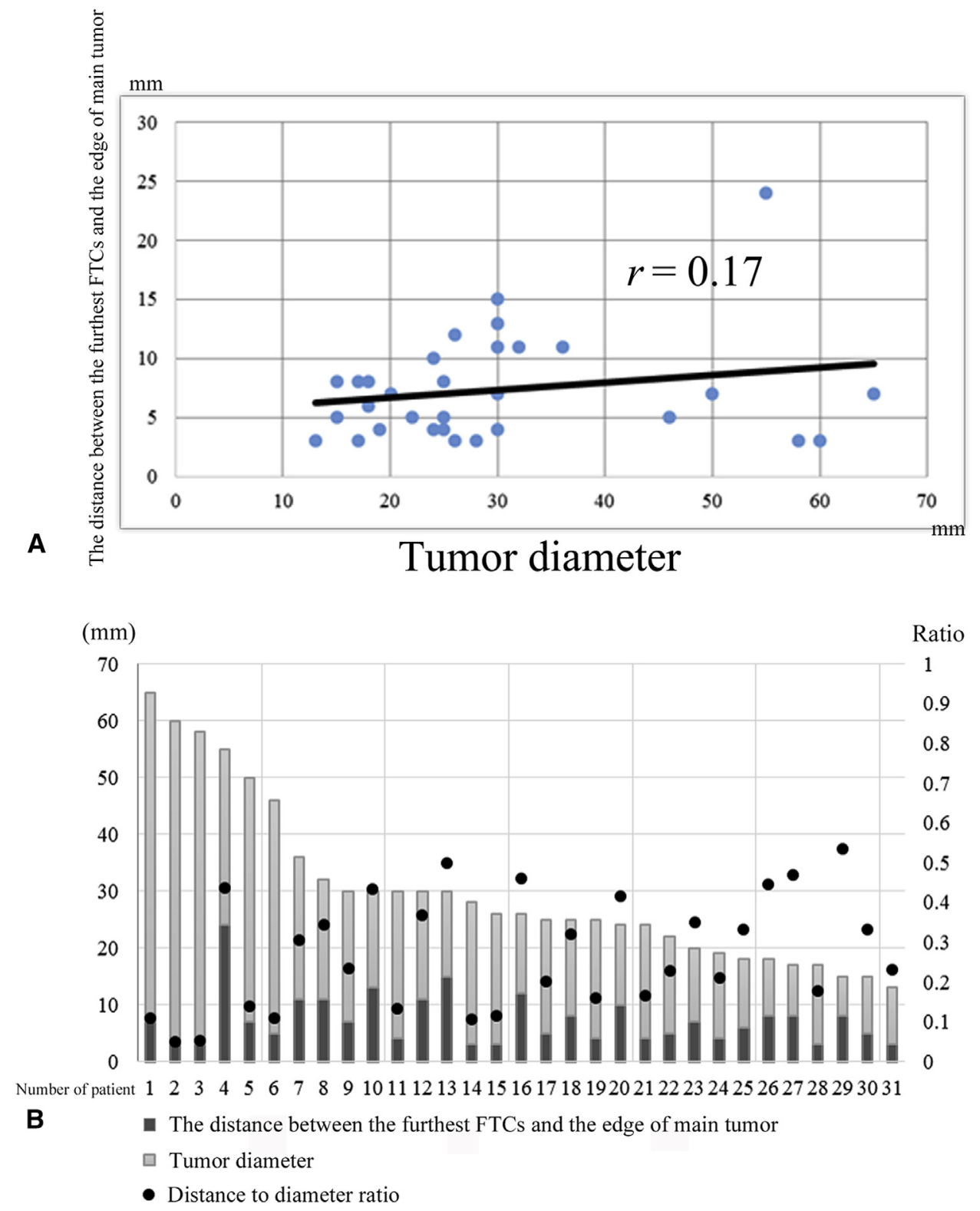

FIGURE 3. Relationship between the diameters of main tumors and the distances of nonintegrated free tumor clusters (FTCS) from the tumor margin. A, Relationship between the diameters of main tumors and the distances of nonintegrated free tumor clusters (FTCs) from the tumor margin. The vertical axis indicates the distance from the tumor edge to the furthest FTC, and the horizontal axis indicates the tumor diameter. B, The vertical axis indicates the ratio of the distance from the tumor edge to the furthest FTC divided by tumor diameter. The horizontal axis shows each patient in the FTC-positive group. All of the ratios were $<1.0$. Therefore, FTCs were observed only within the diameters of the main tumors.

micropapillary subtype of adenocarcinoma. The FTC does not contain solid nests or single cells.

In the present study, 31 of 67 patients with MPCs $(46.2 \%)$ showed FTCs. The current American Joint Committee on Cancer Cancer Staging Manual ${ }^{11}$ notes that microscopic nodules that are not found radiologically or macroscopically are not considered to indicate intrapulmonary metastasis. Hence, the tumor clusters defined in this study were not regarded as intrapulmonary metastasis. The results of the present study suggest a need for modification of pathologic $\mathrm{T}$ staging and the need for discussion of preoperative diagnosis and surgical procedures if the disease is deemed resectable. In addition, diagnosis of MPCs, particularly for FTCs, may be used to select induction therapy before surgery.

Recently, sublobar resection for lung cancer has frequently been used for small peripheral lesions ${ }^{12}$; however, the definition of adequate resection margin remains controversial. According to a retrospective study of 479 patients with $\leq 2$-cm non-small cell lung cancers 

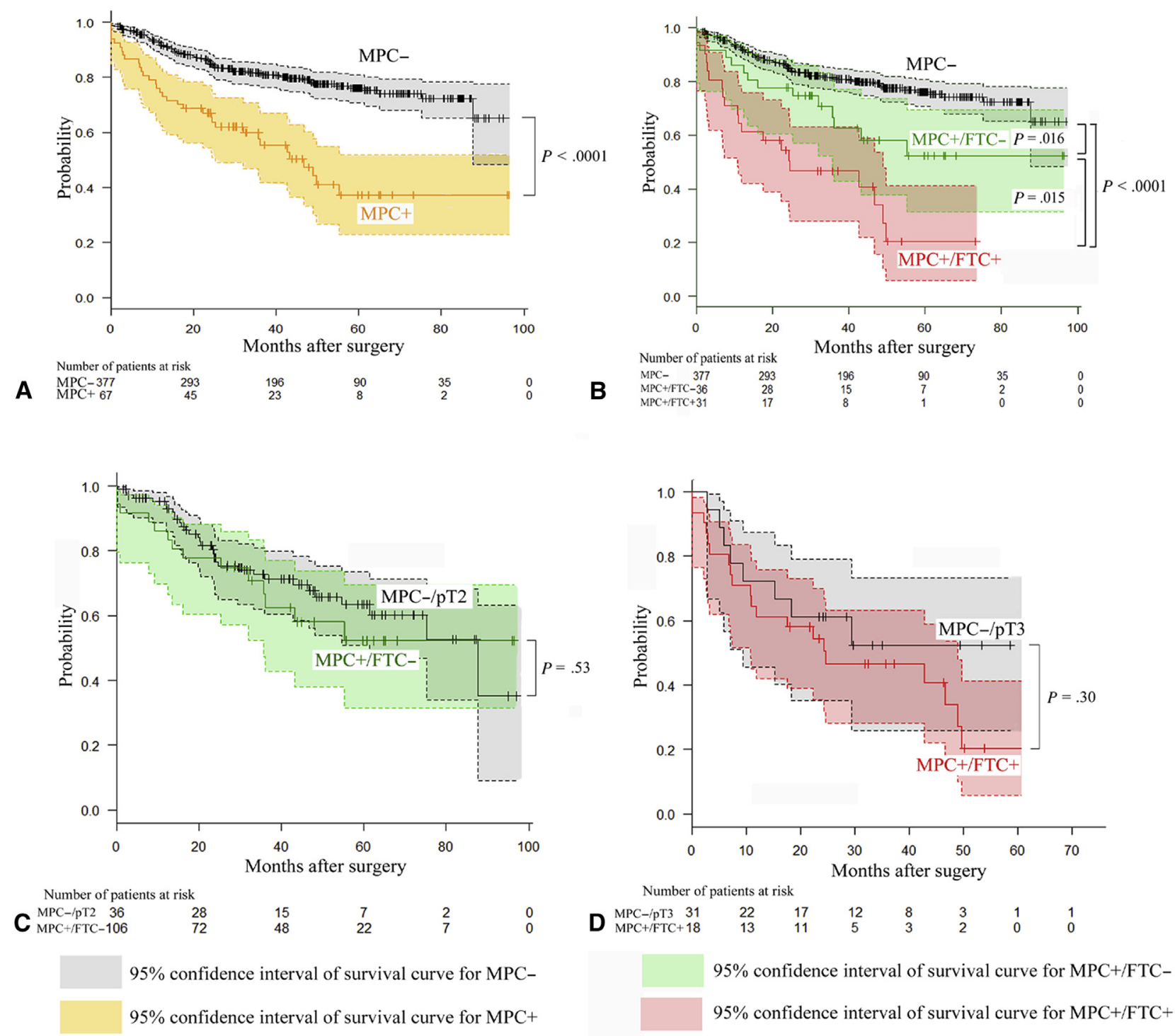

FIGURE 4. Recurrence-free survival curves for each subgroup. A, Patients with MPCs (yellow group) experienced significantly poorer recurrence-free survival compared with patients without MPCs (gray group). B, Both MPC-positive patients without FTCs (green group) and those with FTCs (red group) experienced poorer survival than did patients without MPCs. Patients with FTCs experienced the worst survival among these 3 groups $(P<.001)$. $\mathrm{C}$, Recurrence-free survival curves of patients without MPCs by pT (pathologic assessment of the primary tumor) category. The curve of T2-MPC-negative patients (gray group) was equivalent to that of MPC-positive/FTC-negative patients (green group). D, The curve of T3-MPC-negative patients (gray group) was equivalent to that of MPC-positive/FTC-positive patients (red group). MPC, Micropapillary component; FTC, nonintegrated free tumor cluster.

who underwent wedge resection, ${ }^{13}$ no local recurrences occurred in patients with resection margins $\geq 15 \mathrm{~mm}$. However, Sawabata ${ }^{14}$ reported that the adequate margin depth varies from case to case and that on-site margin cytology is required. Warth et $\mathrm{al}^{15}$ reported that substantial populations $(3.9 \%)$ of micropapillary-predominant subtype tumors were observed even in small (T1a) pulmonary adenocarcinomas, and several reports have indicated that limited surgery is not suitable for patients with MPC, because postoperative recurrence is frequently observed. ${ }^{7,16}$ Our study measured the distance between the furthest edge of FTCs and the margin of the main tumor in each case. We found that all FTCs were observed in an area that did not exceed the diameter of the main tumor. Hence, we suggest that to achieve curative resection, the resection margin should be equal to or exceed the diameter of the main tumor at the time of limited resection, and that this strategy should be used in all cases because FTCs are not recognized by preoperative radiologic imaging or biopsy.

A detailed examination of serial sections found that most tumor clusters that appeared to be separate from the main tumors in each section had continuity with other tumor 
TABLE 3. A multivariate analysis of factors affecting the recurrence-free survival in micropapillary component-negative (gray group) and micropapillary component-positive (yellow group) patients

\begin{tabular}{|c|c|c|c|c|c|}
\hline Characteristics & Variable & Number $(\%)$ & Hazard ratio & $95 \%$ confidence interval & $P$ value \\
\hline \multirow[t]{2}{*}{ Age (y) } & $<64$ & $167(37.6)$ & Reference & & .85 \\
\hline & $\geq 65$ & $277(62.4)$ & 1.15 & $0.78-1.71$ & \\
\hline \multirow[t]{2}{*}{ Surgical procedure } & Limited resection & $126(28.4)$ & Reference & & .089 \\
\hline & Lobectomy or more & $318(71.6)$ & 0.70 & $0.46-1.06$ & \\
\hline \multirow[t]{2}{*}{ Micropapillary components } & Positive & $67(15.1)$ & 2.69 & $1.74-4.16$ & $<.0001$ \\
\hline & Negative & 377 (84.9) & Reference & & \\
\hline \multirow[t]{2}{*}{ Pathologic stage } & Stage I & $338(76.1)$ & Reference & & .0043 \\
\hline & Stage II/III/IV & $106(23.9)$ & 1.98 & $1.24-3.18$ & \\
\hline \multirow[t]{2}{*}{ Adjuvant chemotherapy } & Yes & 347 (78.2) & 0.95 & $0.59-1.53$ & .85 \\
\hline & No & $101(22.8)$ & Reference & & \\
\hline
\end{tabular}

clusters or the main tumor. ${ }^{10}$ Our study showed similar findings: micropapillary clusters were present in approximately the same area within multiple sections. However, some FTCs were also present in small airways containing mucus and appeared to be independent FTCs without any connections. This issue raises a question about whether the coexistence of FTCs represents intrapulmonary metastasis or a part of the main tumor, which influences the decision about the pathologic $\mathrm{T}$ factor. In the present study, disease-free survival of FTC-positive cases was equivalent to that of T3 cases of MPC-negative adenocarcinoma, suggesting that FTCs are likely to have malignant behavior similar to that of intrapulmonary metastases, which primarily spread through the lymphatic system.

The existence of FTCs has appeared to be an important prognostic factor. Onozato et $\mathrm{al}^{16}$ reported the presence of "tumor islands," which were defined as isolated and large collections of tumor cells present within alveolar spaces and that lacked a well-demarcated micropapillary configuration. Tumor islands were observed in $22.2 \%$ of patients with resected early-stage lung adenocarcinomas, and the clinical courses of patients with tumor islands were significantly more likely to mimic those of a predominantly solid pattern and micropapillary pattern. The 5-year recurrence-free survival rates of patients with tumor islands and those without were $44.6 \%$ and $74.4 \%$, respectively. These investigators also found that lung adenocarcinomas with tumor islands were more likely to occur in smokers, had a higher nuclear grade and a solid or micropapillary pattern of growth, and harbored KRAS mutations.

Patients with adenocarcinoma with an MPC, particularly those with MPCs with FTCs, may have the poorest prognosis among all pulmonary adenocarcinoma subtypes and may therefore require different treatment strategies. Hence, how to properly diagnose MPC-positive adenocarcinoma preoperatively remains to be elucidated. To the best of our knowledge, FTCs and similar phenomena have been reported only in association with adenocarcinoma histology. In a previous report, ${ }^{17}$ micropapillary clusters were defined as cohesive clusters of neoplastic cells $(<20$ cells). This study used the same definition for micropapillary clusters. With respect to the number of clusters, only 1 cluster might be observed because of artifacts introduced during the pathologic sectioning process. Hence, we defined the presence of more than 3 clusters as FTCs. In frozen

TABLE 4. A multivariate analysis of factors affecting the recurrence-free survival in micropapillary component-positive/free tumor cluster-negative (green group) and micropapillary component-positive/free tumor cluster-positive (red group) patients

\begin{tabular}{|c|c|c|c|c|c|}
\hline Characteristics & Variation & Number $(\%)$ & Hazard ratio & $95 \%$ confidence interval & $P$ value \\
\hline \multirow[t]{2}{*}{ Age (y) } & $<64$ & $44(65.7)$ & Reference & & .59 \\
\hline & $\geq 65$ & $23(34.3)$ & 1.24 & $0.57-2.70$ & \\
\hline \multirow[t]{2}{*}{ Surgical procedure } & Limited resection & $10(14.9)$ & Reference & & .40 \\
\hline & Lobectomy or more & $57(85.1)$ & 0.60 & $0.19-1.95$ & \\
\hline \multirow[t]{2}{*}{ Surgical margin } & More than tumor diameter & $42(62.7)$ & Reference & & .50 \\
\hline & Less than tumor diameter & $25(37.3)$ & 1.28 & $0.61-2.70$ & \\
\hline \multirow[t]{2}{*}{ Pathologic stage } & I & $33(49.3)$ & Reference & & $<.0001$ \\
\hline & $>\mathrm{II}$ & $34(50.7)$ & 7.75 & $3.17-18.97$ & \\
\hline \multirow[t]{2}{*}{ FTCs } & Positive & $31(46.3)$ & 2.13 & $1.02-4.47$ & .044 \\
\hline & Negative & $36(53.7)$ & Reference & & \\
\hline \multirow[t]{2}{*}{ Adjuvant chemotherapy } & Yes & $24(35.8)$ & 0.61 & $0.289-1.30$ & .20 \\
\hline & No & $43(64.2)$ & Reference & & \\
\hline
\end{tabular}

FTC, Nonintegrated free tumor cluster. 
sections, the lung is not sufficiently inflated. Therefore, it is difficult to diagnose FTCs in the airspace. Currently, the diagnosis of FTCs is possible only in permanent sections, and diagnoses are based on morphologic findings. Thus far, no specific antibodies or biomarkers are available for detecting MPCs or FTCs. We need to identify useful markers for MPCs and FTCs to better select surgical treatment plans for these patients.

A limitation of this study was the retrospective nature. Patients received a variety of postoperative adjuvant therapies. Thus, the survival analysis as well as recurrence pattern analysis might have been affected by these different postsurgical treatments. In addition, the relatively short observation period in some cases may have also affected the results. Another limitation was the small number of patients. The lack of a significant difference in recurrence patterns in patients with MPC between FTC-positive and -negative patients might have been caused by the small number of patients. In addition, the small sample size of the MPC-positive population (including FTC-positive and FTC-negative) for the multivariate analysis may have affected the results of exploring independent prognostic factors. ${ }^{18}$

However, the pathologic sections were all re-reviewed, and a detailed analysis of adenocarcinomas with MPCs was performed. We particularly focused on the existence of FTCs in MPCs and their effect on clinical outcomes. We found that the coexistence of FTCs and MPC-positive adenocarcinomas was associated with a strong negative impact on clinical outcomes of patients who had undergone surgery. In conclusion, the coexistence of FTCs and MPCs was associated with poorer survival in resectable pulmonary adenocarcinomas. Even although the FTCs were nonintegrated tumor cells floating in air spaces, staging might be more accurate if these are treated as pulmonary metastases. The distance between FTCs and the border of the main tumor did not exceed the main tumor diameter.

\section{Conflict of Interest Statement}

Authors have nothing to disclose with regard to commercial support.

\section{References}

1. Travis WD, Brambilla E, Riely GJ. New pathologic classification of lung cancer: relevance for clinical practice and clinical trials. J Clin Oncol. 2012;46:9270
2. Kamiya K, Hayashi Y, Douguchi J, Hashiguchi A, Yamada T, Izumi Y, et al. Histopathological features and prognostic significance of the micropapillary pattern in lung adenocarcinoma. Mod Pathol. 2008;21:992-1001.

3. Zhang Y, Sun Y, Xiang J, Zhang Y, Hu H, Chen H. A clinicopathologic prediction model for postoperative recurrence in stage Ia non-small cell lung cancer. J Thorac Cardiovasc Surg. 2014;148:1193-9.

4. Morales-Oyarvide V, Mino-Kenudson M. High-grade lung adenocarcinomas with micropapillary and/or solid patterns: a review. Curr Opin Pulm Med. 2014;20:317-23.

5. Cha MJ, Lee HY, Lee KS, Jeong JY, Han J, Shim YM, Hwang HS Micropapillary and solid subtypes of invasive lung adenocarcinoma: clinical predictors of histopathology and outcome. Micropapillary and solid subtypes of invasive lung adenocarcinoma: clinical predictors of histopathology and outcome. J Thorac Cardiovasc Surg. 2014;147:921-8.

6. Watanabe M, Yokose T, Tetsukan W, Imai K, Tsuboi M, Ito H, Ishikawa Y, et al. Micropapillary components in a lung adenocarcinoma predict stump recurrence 8 years after resection: A case report. Lung Cancer. 2013;80: 115-234.

7. Kadota K, Nitadori J, Sima CS, Ujiie H, Rizk NP, Jones DR, et al. Tumor spread through air spaces is an important pattern of invasion and impacts the frequency and location of recurrences after limited resection for small stage I lung adenocarcinomas. J Thorac Oncol. 2015;10:806-14.

8. Warth A, Muley T, Kossakowski CA, Goeppert B, Schirmacher P, Dienemann H, Weichert W. Prognostic impact of intra-alveolar tumor spread in pulmonary adenocarcinoma. Am J Surg Pathol. 2015;39:793-801.

9. Van Schil PE, Sihoe AD, Travis WD. Pathologic classification of adenocarcinoma of lung. J Surg Oncol. 2013;108:320-6.

10. Goldstraw P, Crowley J, Chansky K, Giroux DJ, Groome PA, Rami-Porta R, et al. The IASLC Lung Cancer Staging Project: proposals for the revision of the TNM stage groupings in the forthcoming (seventh) edition of the TNM Classification of malignant tumours. J Thorac Oncol. 2007;2: 706-14.

11. AJCC Joint Committee on Cancer. AJCC Cancer Staging Manual. 6th ed. New York: Springer; 2002:167-77.

12. Okada M, Nishio W, Sakamoto T, Uchino K, Yuki T, Nakagawa A, et al. Effect of tumor size on prognosis in patients with non-small cell lung cancer: the role of segmentectomy as a type of lesser resection. J Thorac Cardiovasc Surg. 2005; 129:87-93.

13. Mohiuddin K, Haneuse S, Sofer T, Gill R, Jaklisch MT, Colson YL, et al. Relationship between margin distance and local recurrence among patients undergoing wedge resection for small $(<2 \mathrm{~cm})$ non-small cell lung cancer. J Thorac Cardiovasc Surg. 2014;147:1169-75.

14. Sawabata N. Who should decide margin length in pulmonary excision of lung cancer? J Thorac Cardiovasc Surg. 2014;148:370-1.

15. Warth A, Muley T, Meister M, Stenzinger A, Thomas M, Schirmacher P, et al. The novel histologic International Association for the Study of Lung Cancer/American Thoracic Society/European Respiratory Society classification system of lung adenocarcinoma is a stage-independent predictor of survival. J Clin Oncol. 2012;30:1438-46.

16. Onozato ML, Kovach AE, Yeap BY, Morales-Oyarvide V, Klepeis VE, Tammireddy S, et al. Tumor islands in resected early-stage lung adenocarcinomas are associated with unique clinicopathologic and molecular characteristics and worse prognosis. Am J Surg Pathol. 2013;37: 287-94.

17. Hoshi R, Tsuzuku M, Horai T, Ishikawa Y, Satoh Y. Micropapillary clusters in early-stage lung adenocarcinomas: a distinct cytologic sign of significantly poor prognosis. Cancer. 2004;102:81-6.

18. Peduzzi P, Concato J, Kemper E, Holford TR, Feinstein AR. A simulation study of the number of events per variable in logistic regression analysis. J Clin Epidemiol. 1996;49:1373-9.

Key Words: non-small cell lung cancer, adenocarcinoma, micropapillary pattern, free tumor cluster 


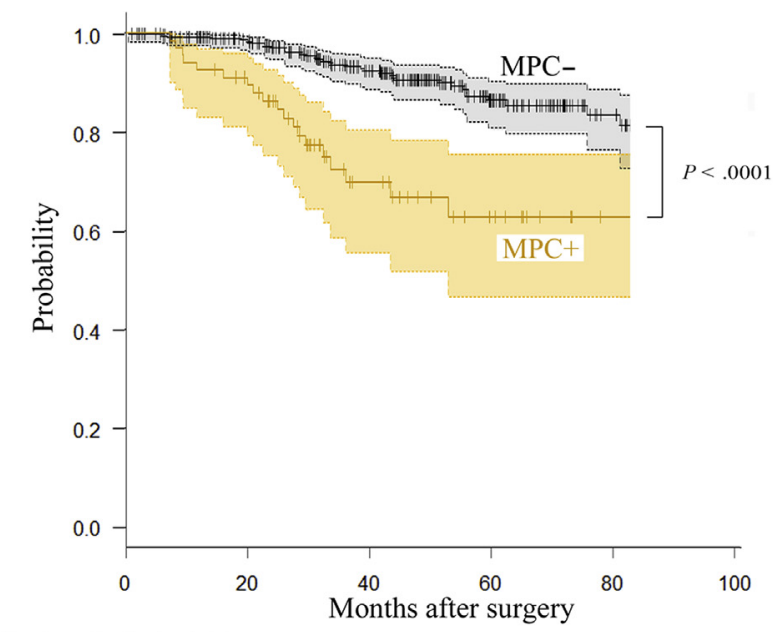

Number of patients at risk

\begin{tabular}{|c|c|}
\hline $\begin{array}{l}\text { MPC+ } 377 \\
\text { MPC- } 67\end{array}$ & $\begin{array}{c}323 \\
57\end{array}$ \\
\hline
\end{tabular}

A

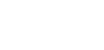

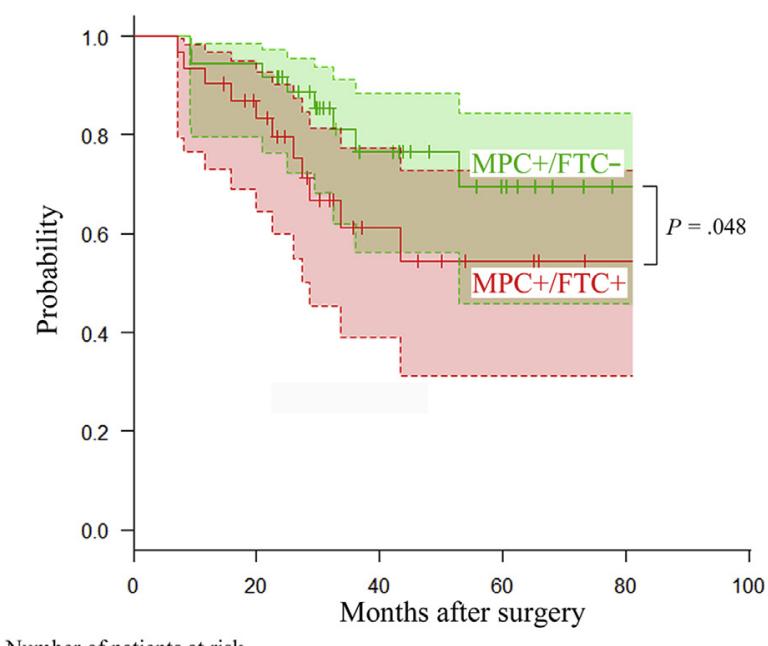

Number of patients at risk

$\begin{array}{llclll}\text { MPC+/FTC- } 36 & 34 & 16 & 8 & 2 & 0 \\ \text { MPC+/FTC+ } 31 & 23 & 9 & 5 & 2 & 0\end{array}$

B

$95 \%$ confidence interval of survival curve for MPC-

$95 \%$ confidence interval of survival curve for MPC +

$95 \%$ confidence interval of survival curve for MPC+/FTC-

$95 \%$ confidence interval of survival curve for MPC+/FTC+

FIGURE E1. Overall survival curves of the patients. A, Patients with micropapillary components (MPCs) experienced significantly poorer overall survival compared with patients without MPCs. B, MPC-positive patients with free tumor clusters experienced poorer survival than those without free tumor clusters.

TABLE E1. 5-year recurrence-free survival rate for Figure 4, $A$ and $B$

\begin{tabular}{|c|c|c|c|}
\hline & MPC- & MPC + & \\
\hline Total $(n=444)$ & $(\mathbf{n}=\mathbf{3 7 7})$ & $(n=67)$ & $P$ value \\
\hline \multirow[t]{2}{*}{ 5-year recurrence-free survival rate } & $76.2 \%(95 \%$ CI, $0.71-0.81)$ & $37.3 \%(95 \%$ CI, $0.23-0.52)$ & $<.0001$ \\
\hline & MPC- & $\mathrm{MPC}+/ \mathrm{FTC}-$ & \\
\hline Total $(n=413)$ & $(\mathbf{n}=\mathbf{3 7 7})$ & $(\mathbf{n}=36)$ & $P$ value \\
\hline \multirow[t]{2}{*}{ 5-year recurrence-free survival rate } & $76.2 \%(95 \%$ CI, $0.71-0.81)$ & $52.2 \%(95 \%$ CI, $0.31-0.70)$ & .016 \\
\hline & MPC- & $\mathrm{MPC}+/ \mathrm{FTC}+$ & \\
\hline Total $(n=407)$ & $(\mathbf{n}=\mathbf{3 7 7})$ & $(\mathbf{n}=\mathbf{3 1})$ & $P$ value \\
\hline \multirow[t]{2}{*}{ 5-year recurrence-free survival rate } & $76.2 \%(95 \%$ CI, $0.71-0.81)$ & $20.4 \%$ (95\% CI, 0.06-0.41) & $<.0001$ \\
\hline & MPC $+/$ FTC + & MPC $+/$ FTC - & \\
\hline Total $(n=67)$ & $(\mathbf{n}=\mathbf{3 1})$ & $(\mathbf{n}=36)$ & $P$ value \\
\hline 5-year recurrence-free survival rate & $20.4 \%(95 \%$ CI, $0.06-0.41)$ & $52.2 \%(95 \%$ CI, $0.31-0.70)$ & .015 \\
\hline
\end{tabular}

$M P C$, Micropapillary component; $F T C$, nonintegrated free tumor cluster. 